\title{
CHARACTERIZATION OF LOCAL, SYSTEMIC AND MUCOSAL IMMUNE RESPONSES AFTER ORAL DNA VACCINATION AGAINST SPRING VIREMIA OF CARP VIRUS
}

\author{
Carmen WE Embregts*», Geert F Wiegertjes, Maria Forlenza* \\ Cell Biology and Immunology Group, Wageningen University, the Netherlands
}

DNA vaccination through i.m. injection of DNA plasmid encoding the G protein of SVCV (SVCV-G) is very successful in carp but this method of vaccine delivery is not practical. Alternatively, oral vaccination would be highly preferred due to its stress-free, labour-extensive and easy delivery. On the downside, oral vaccines are often less efficacious when compared to traditional injection vaccines and little is known about their activation of immune mechanisms. In an effort to generate an oral DNA vaccine against SVCV, carp of $2 \mathrm{~g}$ were orally vaccinated three times with alginate microspheres containing either 8 ug pcDNA3 plasmid, 8 ug pcDNA3-SVCVG plasmid, or PBS. Owing to the availability of several carp leukocyte-specific monoclonal antibodies, we revealed the early recruitment of specific populations of leukocytes to the intestine and spleen following oral vaccination. The combined use of cell type-specific antibodies and an antibody against the SVCV-G protein allowed us for the first time, to identify the sites and kinetics of expression of the SVCV-G protein, and to concomitantly recognize the cell types involved in antigen uptake and processing.

The use of a second panel of antibodies enabled us to distinguish between various populations of both $\mathrm{B}$ and $\mathrm{T}$ lymphocytes, allowing the study of their specific roles during vaccination and infection. Using this panel we were able to characterise kinetics of recruitment of specific B and T lymphocyte populations to local sites after vaccination, as well as their distribution in systemic and other mucosal immune organs. Besides the characterization of local and systemic immune responses after oral DNA vaccination, using a wide array of techniques including RT-qPCR, histology, flow cytometry and in vitro macrophage-T cell cultures, we are currently studying the induction of immune memory after oral vaccination. This combination of techniques enables us to establish an unique overview of the kinetics of the immune response of carp after oral DNA vaccination against SVCV, at cell, protein and gene expression level and in a broad selection of immune-relevant organs. Together we will present the promising potential of an oral DNA vaccine against SVCV to not only induce an early local immune response, but also a systemic responses followed by long-term memory formation.

\section{KEYWORDS}

DNA vaccination, SVCV, oral vaccination, $\mathrm{T}$ lymphocytes, immune memory

"These authors have contributed equally to this work.

${ }^{\S}$ Corresponding author. Tel.: +31 317483708; Fax: +31 317483962.

E-mail address: carmen.embregts@wur.nl 